\title{
Fine-tuning of the optical properties of hollow-core light cages using dielectric nanofilms
}

\author{
Bumjoon Jang ${ }^{1}$, Julian Gargiulo ${ }^{2}$, Mario Ziegler $^{1}$, Ron Fatobene Ando ${ }^{1}$, Uwe Hübner ${ }^{1}$, \\ Stefan A. MaieR ${ }^{2,3}$, And Markus A. Schmidt ${ }^{1,4,5, *}$ \\ ${ }^{1}$ Leibniz Institute of Photonic Technology, Albert-Einstein-Str. 9, 07745 Jena, Germany \\ ${ }^{2}$ Chair in Hybrid Nanosystems, Nanoinstitute Munich, Königinstrasse 10, Ludwig-Maximilians-Universität Munich, 80539 Munich, Germany \\ ${ }^{3}$ Department of Physics, Imperial College London, London SW7 2AZ \\ ${ }^{4}$ Otto Schott Institute of Materials Research (OSIM), Friedrich Schiller University of Jena, Fraunhoferstr. 6, 07743 Jena, Germany \\ ${ }^{5}$ Abbe Center of Photonics and Faculty of Physics, Friedrich-Schiller-University Jena, Max-Wien-Platz 1, 07743 Jena, Germany \\ *Corresponding author: markus.schmidt@leibniz-ipht.de
}

Compiled October 30, 2019

Here we show that the optical properties of direct-laserwritten on-chip hollow-core waveguides - so-called light cages - can be controlled to a very high degree by dielectric nanofilms. Using low-temperature atomic layer deposition (ALD), alumina nanofilms were concentrically deposited on the high-aspect strands which surround the central air core and confine the light via the anti-resonant effect. In accordance with modal cut-off simulations without any free parameters, a linear spectral shift of the resonances with increasing film thickness is experimentally observed. The phenomenon is explained by a shift of the dispersions of cladding supermodes. As neither cage geometry nor polymer is affected by the film deposition our results suggest atomic layer deposition to be an essential tool for fine-tuning the properties of hollow core light cages and to protect them from aggressive substances, being relevant for, e.g., bioanalytics or quantum technology. (C) 2019 Optical Society of America

\section{http://dx.doi.org/10.1364/ao.XX.XXXXXX}

Precise tailoring of the optical properties of waveguides is relevant for many applications that demand accurate control of modal dispersion (e.g., nonlinear frequency conversion [1, 2]) or on the spectral domains of operation [3]. For instance, hollow core waveguides $[4,5]$ show distinct resonances in the transmission spectra [5] due to microstructured claddings employed. Locating the target wavelength into a high-transmission spectral domain is crucial for many applications while, however, fabrication cannot achieve the required level of precision in many situations demanding an additional post-processing step.

One highly attractive methodology for post-fabrication tuning is the deposition of dielectric nano-films via atomic layer deposition (ALD) [6]. Monolayers can be successively deposited across almost any kind of surface shape in a conformal way. This allows for a unique control on the film thickness with angstrom precision, yielding an extraordinarily high potential for tuning the properties of photonic structures, examples of application areas include nonlinear optics [7] and optical microresonators [8]. Nano-films have also been used for protecting photonic structures from corrosive materials (e.g., alkali vapor [9]).

Within planar waveguide technology, the most widely employed hollow core waveguide is the antiresonant reflecting optical waveguide (ARROW) relying on dielectric multilayers encapsulating a central hollow core [10]. Even though being successfully used in bioanalytics [11] and atomic spectroscopy [12], fine-tuning of this device is rather difficult. As materials can enter the core region only via the open ends, ARROW is incompatible with ALD due to long gas exchange times. (a) nano-film
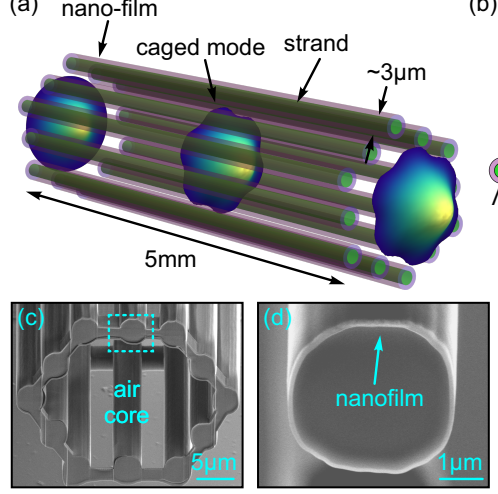

(b)
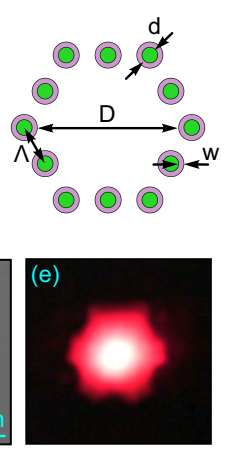

Fig. 1. (a) Sketch of the hollow-core light cage that has been coated by nano-films using atomic layer deposition. (b) Sketch including all relevant parameters. (c) Scanning electron micrographic (SEM) image of a coated light cage (center-to-center distance (pitch) $\Lambda=7 \mu \mathrm{m}$, strand diameter $d=3.6 \mu \mathrm{m}$, nanofilm thickness $w=100 \mathrm{~nm}$ ). (d) Close-up view of the strand highlighted in (c). Note that for both SEM images the sample has been prepared by focused ion beam milling to make the nano-film visible. (e) Example measured mode field pattern $(\lambda$ $=625 \mathrm{~nm}$ ).

A novel type of on-chip hollow core waveguide - the light 
cage - that allows for the side-wise introduction of surrounding material, for gasses in particular, was recently introduced (Fig. 1 (a)) [13]. This geometry consists of freely suspended high-aspect-ratio dielectric strands surrounding a hollow core. The modes of the strands form cladding supermodes at certain frequencies which are in anti-resonance with the central core mode [14]. Due to its unique shape, this device yields various key features such as direct access to the core region through the transversally separated strands, high fraction of modal fields in the hollow core section, and mode propagation over centimeter distances. First gas sensing experiments have been performed [13], while a photonic band gap version of the light cage with significantly reduced modal attenuation at small core sizes was recently reported [15].

The implementation of the light cage geometry relies on direct-laser-writing (DLW), an approach that allows for straightforward access to all three spatial dimensions. Photonic structures such as sophisticated fiber-integrated lenses [16] or waveguide interconnects [17] have been implemented. Within the context of ALD-deposited nano-films, DLW has been used for laminate optical metamaterials [18], multifunctional materials [19] and woodpile photonic crystals [20].

In this work, we show that ALD can be used for the tailoring of the optical properties of light cages at extremely high levels of accuracy. We conformally coated the surfaces of light cages by high refractive index (RI) nano-layers of $\mathrm{Al}_{2} \mathrm{O}_{3}$ of defined thickness using low-temperature (low-T) ALD. As shown in experiments and simulation, the nano-layer has a significant impact on the dispersions of the various modes. This leads to a substantial modification of the transmission of the fundamental core mode, i.e., to a spectral shift of the resonances.

The transverse arrangement of a twelve strand light cage is shown in Figs. 1(a) and (b). The formation of a defect core mode is provided by the anti-resonant effect and the light guidance relies on inhibited coupling of the core mode to the supermodes formed in the cladding [21]. As a result, this geometry allows the light to propagate over millimeters in a comparably sparse and transversely open structure [13].

A characteristic feature associated with anti-resonant guidance are strong dips in the spectral distribution of the core mode transmission resulting from an avoided crossing of the dispersions of core mode and cladding supermodes and leading to transverse energy dissipation [14, 22]. The spectral position of these resonances $\lambda_{R}$ is strongly correlated to the dispersion of the supermodes which are determined by the isolated waveguides modes. As a consequence, any change to the geometry of a single strand will affect their dispersions and therefore the overall transmission properties of the light cage. Within this work, modal dispersion is modified by coating concentric high RI nano-layers conformally around the strands, leading to distinct spectral shifts of the resonances.

To qualitatively reveal the impact of the nano-layers, we calculate the dispersion of one selected supermode band while varying layer thickness $w$ at fixed nano-layer RI assuming an air environment $\left(n_{\text {air }}=1\right)$. Specifically, we calculate the spectral distribution of the edges of the $\mathrm{LP}_{05}$-band (isolated LP-mode of fifth radial order) which allows both identifying the regions of non-zero density of states as well as finding the wavelengths of the resonances [23]. A simplified version of this scalar-wave approach has been successfully employed for the analysis of the band structure of various types of band gap fibers [24-26] and single-ring anti-resonant hollow core fibers [27]. All reported results have shown that the optical properties of the mentioned
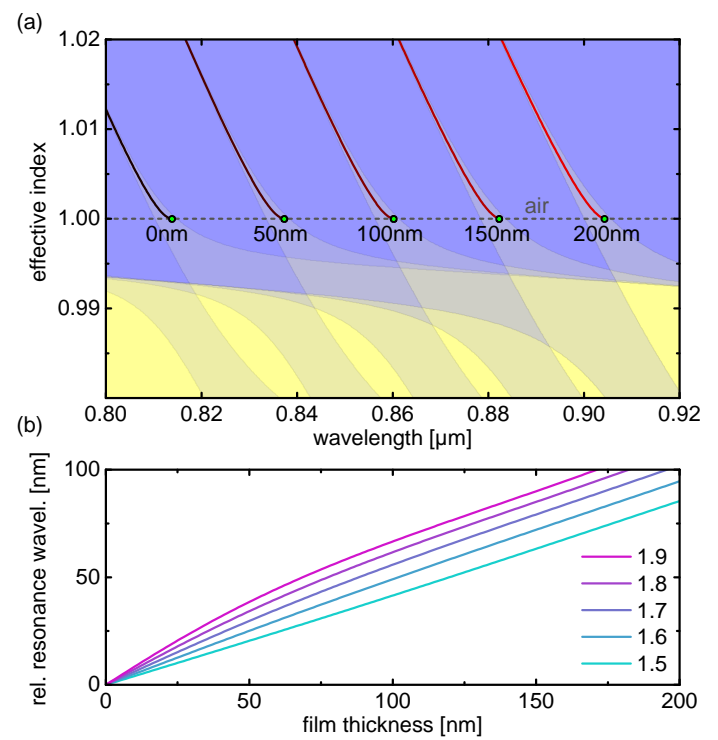

Fig. 2. (a) Dispersion of one supermode band for different thicknesses of the nano-film (thickness indicated by the numbers, $\mathrm{LP}_{05}$-mode). The light yellow background refers to the region of non-zero density of cladding states, while there are no photonic states in the blue domains. The reddish lines show the dispersions of the respective isolated modes that cut-off at the index of air (indicated by the green dots). (b) Relative resonance wavelength $\Delta \lambda=\lambda_{R}-\lambda_{R}(t=0)$ as a function of film thickness for five different constant RIs of the nano-layer (legend indicates RI). Simulations include the full polymer dispersion and assume air as surrounding medium.

waveguides are dominated by the modes with the two lowest radial orders $(l=0$ and $l=1)$, which emerge as dips (i.e., resonances) in the transmission spectrum of the central core mode. In this work the RI of the strand material (polymer) has been included using a recently reported Sellmeier model [15] while the $\mathrm{RI}$ of $\mathrm{Al}_{2} \mathrm{O}_{3}$ has been determined by ellipsometry (defined in Appendix B). As shown in Fig. 2(a) $(d=3.64 \mu \mathrm{m}, \Lambda=7 \mu \mathrm{m})$, an increasing nano-layer thickness imposes the band to red-shift, which can be explained by a corresponding shift of the dispersion of the associated isolated mode (reddish colored curves in Fig. 2) that increasingly probe the high RI material.

To compare the experimentally measured resonance wavelengths $\lambda_{R}$ to simulations, we define the simulated resonance as being identical to the cut-off wavelengths of the isolated mode $\lambda_{c}$ which is defined by the condition $n_{\text {eff }}=1$ (green dots in Fig. 2) [15]. The dependence of $\lambda_{c}$ on the various parameters can be straightforwardly revealed by assuming a scalar wave function ansatz within the concentric three layer system, leading to a quasi-analytical equation (Eq. 1, Appendix A). Numerical analysis of one selected resonance $\left(\lambda_{R}(t=0)=0.815 \mu \mathrm{m}\right.$, $d=3.64 \mu \mathrm{m}, \mathrm{LP}_{05}$-mode) shows that for constant core parameters and film thicknesses $<200 \mathrm{~nm}$, RIs of the layer below 1.7 lead to a linear shift of the relative resonance wavelength $\Delta \lambda_{c}=\lambda_{c}(t)-\lambda_{c}(t=0)$ (Fig. 2(b)), while a nonlinear dependency is observed for higher RIs.

The uncoated light cages were fabricated via a commercial two-photon lithography device (Photonic Professional GT, Nanoscribe $\mathrm{GmbH}$ ). Utilizing the three-dimensional (3D) nature of the DLW technique, complex 3D light cages were polymerized using 
a liquid negative-tone photo-resin (IP-Dip, Nanoscribe GmbH). The development process removes the unsolidified resin leaving the final structure on a silicon substrate (fabrication details can be found in [13]). The resulting light cages (pitch $\Lambda=7$ $\mu \mathrm{m}$, strand diameter $d \approx 3.6 \mu \mathrm{m}$ ) have lengths of $5 \mathrm{~mm}$. The achieved aspect ratio of the strands is as large as $\approx 1000$ overall defining a waveguide geometry with significantly higher aspect ratios compared to other resonant structures [28].

After fabrication, the light cage were coated by conformal nano-layers using low-T ALD. The low temperatures were necessary to avoid degradation of the light cages due to elevated process temperatures. Specifically, we used plasma-enhanced ALD (PE-ALD) to achieve a conformal coating of alumina at a very low substrate temperature of only $30^{\circ} \mathrm{C}$ (SEM image of an example of a coated light cage is shown in Fig. 1(d)). The PE-ALD process was defined by two self-terminating single surface half reactions using Trimethylaluminum (TMA) and oxygen plasma as precursors. The growth rate was 2.2 angstrom/per cycle [29]. To implement samples with different film thicknesses, only the number of cycles was adjusted to the growth per cycle. Here, we deposited alumina films with thicknesses of 50, 100, 150 and $200 \mathrm{~nm}$. The film thickness was verified on a separate silicon chip using ellipsometry.

Interestingly, the oxygen plasma did not cause any observable alteration of the polymer structure, which was due to the remote inductively coupled plasma configuration of our ALD setup. The remote configuration led to diminished number of reactive species onto the substrate surface and in turn, avoided the etching of the polymer cages during plasma exposure [30].

The optical setup to characterize the transmission through the light cages includes a combination of a broadband light source (SuperK COMPACT, NKT Photonics, $450 \mathrm{~nm}<\lambda<2400$ $\mathrm{nm})$, coupling optics and spectral diagnostics. The collimated, unpolarized white light was focused onto one end of light cage by a 10x microscope objective to excite the core mode. A notch filter centered at $1065 \mathrm{~nm}$ (FWHM = $73 \mathrm{~nm}$, Edmund Optics) was applied to block residual pump light. The transmitted light was collected by another 10x objective and fed into an optical spectrum analyser (AQ-6315A, Ando) by a multimode fiber (FG050LGA, core size $50 \mu \mathrm{m}, \mathrm{NA}=0.22$ ). The transmitted mode profile was monitored by a CCD camera. Polarization control was not relevant for the experiments.

The optical characterization shows clear transmission within the fundamental core mode (example mode image shown in Fig. 1(e)) between $450 \mathrm{~nm}$ and $1.2 \mu \mathrm{m}$ with resonances at characteristic wavelengths (Fig. 3(b)). The corresponding simulated cut-off wavelengths $\lambda_{c}$ (dots in the light green area in Fig. 3(a)) match to the measured dip positions $\lambda_{R}$ (green dots in Fig. 3(b)) to a very high degree. This shows the high quality of the fabricated structure and the elaborate control on the optical properties provided by DLW. As suggested by the modal simulations (Fig. 2), the experiments clearly reveal that increasing the thickness of the nano-film leads to an increase of $\lambda_{R}$, which (as suggested by Fig. 2(a)) is a result of the shift of isolated mode dispersions. It is important to note that as shown in Fig. 3(a), the experimentally determined spectral positions of the resonances and the overall linear spectral shifts (dots in Fig. 3(a)) quantitatively match simulations (using Eq. 1 and the material dispersion stated above). This is remarkable given the fact that the simulations do not include any free parameter (solid colored lines in Fig. $3(\mathrm{a})$ ). The agreement is even more evident when fitting a linear dependence to the experimental data (dashed colored lines in Fig. 3(a)) showing hardly any difference to the simulations

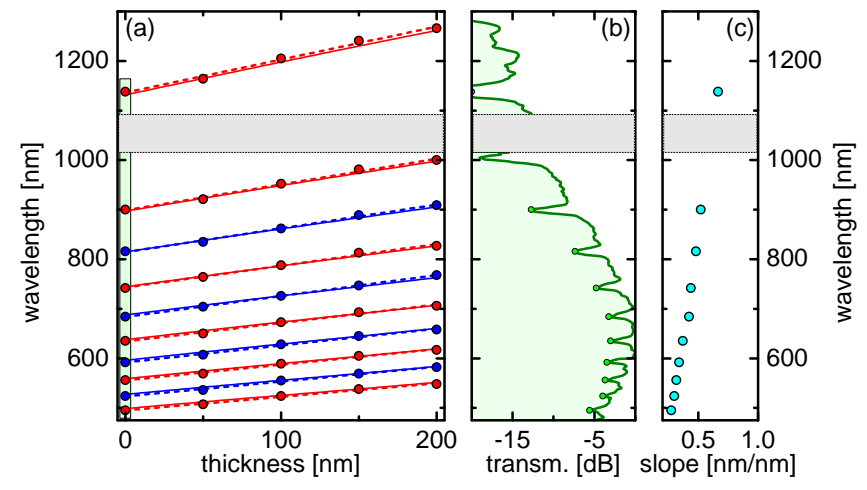

Fig. 3. (a) Measured resonance wavelengths (dip wavelength) as function of nano-film thickness (dots) together with simulated data (solid lines) and linear fits (dashed lines). The two colors refer to different azimuthal mode orders (blue: $l=0$, red: $l=1$ ). (b) Spectral distribution of the transmission for the uncoated sample $(t=0)$. The green dots indicate the spectral positions of the resonances. (c) Slope of the linear fits shown in (a) for the different resonances. To identify the individual resonance, each slope value is plotted at the resonance wavelength of the uncoated sample (within the light green area in (a)).

(solid lines in Fig. 3(a)). This fact reveals that low-T ALD neither impacts the light cage geometry nor the polymer, making this approach a valuable tool to fine-tune the optical properties of DLW-implemented 3D structures.

The magnitude of the spectral shift (i.e., the tuning slope $S_{t}$ ) can be quantified by the slope of the linear fits for each resonance independently $\left(S_{t}=\partial \lambda_{R} / \partial w\right)$, showing values of the order of $0.5 \mathrm{~nm} / \mathrm{nm}$ for all resonances. Therefore, a $200 \mathrm{~nm}$ nanolayer imposes the resonances to shift by about $100 \mathrm{~nm}$, which at least for the short wavelength resonances is more than the spectral distances between the resonances in the uncoated situation. Conversely, the angstrom precision provided by ALD allows to spectrally tune the resonance at the sub-nm level, representing an accuracy that cannot be achieved by DLW fabrication alone. At this stage it is important to note that the tuning slope has a negligible dependence on strand diameter, which was shown on the basis of a trial simulation on the example of the $\mathrm{LP}_{06}$-mode showing that the change of $S_{t}$ within the strand diameter interval $3.6 \mu \mathrm{m}<d<3.7 \mu \mathrm{m}$ is smaller than $0.13 \%$. The assumed diameter variation of $100 \mathrm{~nm}$ is actually much larger compared to what can expected in experiments. Here a statistical analysis of measured $\lambda_{R}$ of a series of coated light cages has shown very low spectral variation for three selected modes $\left(\mathrm{LP}_{17^{-}}, \mathrm{LP}_{06}{ }^{-}\right.$, $\mathrm{LP}_{16}$-mode), since the calculated standard deviations are very small $\left(\sigma_{\lambda_{R}}<1 \mathrm{~nm}\right)$. This quantity can be straightforwardly translated into a corresponding standard deviation of the strand diameter $\sigma_{d}$ via the cut-off condition of weakly guided modes [31] . Here a preliminary study has shown $\sigma_{d}<5 \mathrm{~nm}$ for all three modes, emphasizing the high quality of the structures produced. These two findings are essential for the concept presented here, since regardless of nano-film thickness the resonances practically shift by the same amount at identical tuning slope.

Fine-tuning the optical properties of in particular hollow core waveguides within a post-processing step is essential for many applications due to implementation inaccuracies and resonances in the transmission spectra. Here we show that the resonances of DLW-implemented light cages can be controlled to a very 
high degree by dielectric nano-films deposited on the strands. Specifically, the use of low-T ALD allows for the conformal coating of the strands of light cages with $\mathrm{Al}_{2} \mathrm{O}_{3}$ layer with subnanometer precision. This leads to a linear spectral shift of the resonance confirmed by a cut-off model that include no free parameter. The results indicate that neither the cage geometry nor the polymer material are affected by the ALD process and that high-quality nano-layers with defined properties have been deposited on the strands. Such fine-tuning capability is relevant for applications that demand precise control on dispersion such as ultrashort pulse propagation [32] or nonlinear light generation [33] or on the spectral domain of operation (e.g., atomic vapor spectroscopy [34]). Moreover, the deposited nano-films can be used as chemically active surfaces for, e.g., catalysis [35, 36] or for improving biocompatibility [37]. Moreover the nanofilms protect the light cages from aggressive substances which is important in, e.g., alkali vapor based spectroscopy [9] or liquid sensing applications.

\section{APPENDIX: CUTOFF SIMULATIONS}

The scalar wave function of the three-layer system (polymer$\mathrm{Al}_{2} \mathrm{O}_{3}$-air) is as follows:

$$
\psi(r)= \begin{cases}A_{1} \cdot J_{l}\left(\kappa_{1} r\right) & n(r)=n_{1}, r \leq a \\ A_{2} \cdot J_{l}\left(\kappa_{2} r\right)+B_{2} \cdot Y_{l}\left(\kappa_{2} r\right) & n(r)=n_{2}, a<r \leq b \\ A_{3} \cdot(r / b)^{-l} & n(r)=n_{3}, b<r\end{cases}
$$

where $a=d / 2$ and $b=a+w\left(A_{\mathrm{i}}\right.$ : constants). The transverse wave vectors in the first two layers at cutoff $\left(n_{\text {eff }}=n_{3}\right)$ are given by $\kappa_{1}=k_{0}\left(n_{1}^{2}-n_{3}^{2}\right)^{1 / 2}$ and $\kappa_{2}=k_{0}\left(n_{2}^{2}-n_{3}^{2}\right)^{1 / 2}$, where $k_{0}=$ $2 \pi / \lambda_{0}$. Assuming continuity of $\psi(r)$ and $\psi^{\prime}(r)$ at $r=a$ and $r=b$ yields the dispersion equation:

$$
\frac{Y_{l-1}\left(\kappa_{2} b\right)}{J_{l-1}\left(\kappa_{2} b\right)}=\frac{\kappa_{1} Y_{l}\left(\kappa_{2} a\right) J_{l+1}\left(\kappa_{1} a\right)-\kappa_{2} J_{l}\left(\kappa_{1} a\right) Y_{l+1}\left(\kappa_{2} a\right)}{\kappa_{1} J_{l}\left(\kappa_{2} a\right) J_{l+1}\left(\kappa_{1} a\right)-\kappa_{2} J_{l}\left(\kappa_{1} a\right) J_{l+1}\left(\kappa_{2} a\right)}
$$

The RI of $\mathrm{Al}_{2} \mathrm{O}_{3}$ was fitted using $n_{\mathrm{Al}_{2} \mathrm{O}_{3}}(\lambda)=B_{0}+B_{1} / \lambda^{2}$ with $B_{0}=1.57072$ and $B_{1}=5.54962 \cdot 10^{-3} \mathrm{~nm}^{2}$.

\section{FUNDING INFORMATION}

German Research Foundation via grant No. SCHM2655/8-1, Bavarian Solar Technologies Go Hybrid (SolTech) programme

\section{ACKNOWLEDGMENT}

S.A.M. additionally acknowledges the Lee-Lucas Chair in Physics. J. G. acknowledges funding from the European Commission for the Marie-Sklodowska-Curie action 797044.

\section{REFERENCES}

1. M. Belal, L. Xu, P. Horak, L. Shen, X. Feng, M. Ettabib, D. J. Richardson, P. Petropoulos, and J. H. V. Price, Opt. Lett. 40, 2237 (2015).

2. M. Chemnitz, R. Scheibinger, C. Gaida, M. Gebhardt, F. Stutzki, S. Pumpe, J. Kobelke, A. Tünnermann, J. Limpert, and M. A. Schmidt, Optica. 5, 695 (2018).

3. F. Benabid, J. C. Knight, G. Antonopoulos, and P. S. Russell, Science. 298, 399 (2002).

4. D. Yin, H. Schmidt, J. P. Barber, and A. R. Hawkins, Opt. Express 12, 2710 (2004)

5. F. Couny, F. Benabid, and P. S. Light, Opt. Lett. 31, 3574 (2006).

6. V. Miikkulainen, M. Leskela, M. Ritala, and R. L. Puurunen, J. Appl. Phys. 113 (2013).
7. S. C. Warren-Smith, M. Chemnitz, H. Schneidewind, R. Kostecki, H. Ebendorff-Heidepriem, T. M. Monro, and M. A. Schmidt, Opt. Lett. 42, 1812 (2017).

8. J. Riemensberger, K. Hartinger, T. Herr, V. Brasch, R. Holzwarth, and T. J. Kippenberg, Opt. Express 20, 27661 (2012).

9. R. Ritter, N. Gruhler, W. H. P. Pernice, H. Kübler, T. Pfau, and R. Löw, New J. Phys. 18, 103031 (2016).

10. D. Yin, J. P. Barber, A. R. Hawkins, and H. Schmidt, Opt. Express 13, 9331 (2005).

11. G. Testa, Y. Huang, P. M. Sarro, L. Zeni, and R. Bernini, Opt. Lett. 35, 1584 (2010).

12. W. Yang, D. B. Conkey, B. Wu, D. Yin, A. R. Hawkins, and H. Schmidt, Nat. Photonics 1, 331 (2007).

13. C. Jain, A. Braun, J. Gargiulo, B. Jang, G. Li, H. Lehmann, S. A. Maier, and M. A. Schmidt, ACS Photonics 6, 649 (2019).

14. M. Zeisberger and M. A. Schmidt, Sci. Reports 7, 11761 (2017).

15. B. Jang, J. Gargiulo, R. F. Ando, A. Lauri, S. A. Maier, and M. A. Schmidt, Opt. Lett. 44, 4016 (2019).

16. T. Gissibl, S. Thiele, A. Herkommer, and H. Giessen, Nat. Photonics 10, 554 (2016).

17. P. I. Dietrich, M. Blaicher, I. Reuter, M. Billah, T. Hoose, A. Hofmann, C. Caer, R. Dangel, B. Offrein, U. Troppenz, M. Moehrle, W. Freude, and C. Koos, Nat. Photonics 12, 241 (2018).

18. A. Wickberg, C. Kieninger, C. Sürgers, S. Schlabach, X. Mu, C. Koos, and M. Wegener, Adv. Opt. Mater. 4, 1203 (2016).

19. N. G. Dou, R. A. Jagt, C. M. Portela, J. R. Greer, and A. J. Minnich, Nano Lett. 18, 4755 (2018). PMID: 30022671.

20. C. Marichy, N. Muller, L. S. Froufe-Pérez, and F. Scheffold, Sci. Reports 6, 21818 (2016).

21. B. Debord, A. Amsanpally, M. Chafer, A. Baz, M. Maurel, J. M. Blondy, E. Hugonnot, F. Scol, L. Vincetti, F. Gérôme, and F. Benabid, Optica 4, 209 (2017).

22. A. Hartung, J. Kobelke, A. Schwuchow, J. Bierlich, J. Popp, M. A. Schmidt, and T. Frosch, Opt. Lett. 40, 3432 (2015).

23. T. A. Birks, G. J. Pearce, and D. M. Bird, Opt. Express 14, 9483 (2006).

24. M. A. Schmidt, N. Granzow, N. Da, M. Peng, L. Wondraczek, and P. S. Russell, Opt. Lett. 34, 1946 (2009).

25. G. Li, M. Zeisberger, and M. A. Schmidt, Opt. Express 25, 22467 (2017).

26. J. M. Stone, G. J. Pearce, F. Luan, T. A. Birks, J. C. Knight, A. K. George, and D. M. Bird, Opt. Express 14, 6291 (2006).

27. R. F. Ando, A. Hartung, B. Jang, and M. A. Schmidt, Opt. Express 27, 10009 (2019).

28. C. Sieutat, R. Peretti, J.-L. Leclercq, P. Viktorovitch, and X. Letartre, Opt. Express 21, 20015 (2013).

29. S. Yüksel, M. Ziegler, S. Goerke, U. Hübner, K. Pollok, F. Langenhorst, K. Weber, D. Cialla-May, and J. Popp, The J. Phys. Chem. C 119, 13791 (2015).

30. M. Ziegler, L. Fritzsch, J. Day, S. Linzen, S. Anders, J. Toussaint, and H.-G. Meyer, Supercond. Sci. Technol. 26, 025008 (2012).

31. A. Snyder and J. Love, Optical Waveguide Theory (Springer, Boston, MA, 1983).

32. J. C. Travers, J. M. Stone, A. B. Rulkov, B. A. Cumberland, A. K. George, S. V. Popov, J. C. Knight, and J. R. Taylor, Opt. Express 15, 13203 (2007).

33. R. Sollapur, D. Kartashov, M. Zürch, A. Hoffmann, T. Grigorova, G. Sauer, A. Hartung, A. Schuchow, J. Bierlich, J. Kobelke, M. Chemnitz, M. A. Schmidt, and C. Spielmann, Light. Sci. \& Appl. 6, e17124 (2017).

34. H. Schmidt and A. R. Hawkins, Laser \& Photonics Rev. 4, 720 (2010).

35. V. Pore, A. Rahtu, M. Leskelä, M. Ritala, T. Sajavaara, and J. Keinonen, Chem. Vap. Deposition 10, 143 (2004).

36. A. M. Cubillas, S. Unterkofler, T. G. Euser, B. J. M. Etzold, A. C. Jones, P. J. Sadler, P. Wasserscheid, and P. S. Russell, Chem. Soc. Rev. 42, 8629 (2013)

37. M. Putkonen, T. Sajavaara, P. Rahkila, L. Xu, S. Cheng, L. Niinistö, and H. J. Whitlow, Thin Solid Films 517, 5819 (2009). 